\title{
A model for change
}

\section{Alison Williams \& Judy Robertson}

We are what we repeatedly do.

Excellence then is not an act but a habit.

(Aristotle)

If a university has decided to adopt gender equality as a goal, how should it go about achieving it? It is not easy; it is not a single problem to solve. Various factors interact to produce inequitable and inhospitable working environments including overt discrimination and harassment, societal stereotypes, unconscious bias, and promotion and hiring procedures which promote or fail to protect against bias. Gender inequalities affect different people in different ways. Some people may struggle as a result of working under stereotype threat - a well-researched psychological phenomenon where an individual's performance and confidence on a task is reduced by being a member of a minority group which has negative stereotypes associated with that task.

Others may be held back by anxiety stemming from imposter syndrome which is the result of feeling as if one does not belong in an academic environment. Even if a working environment is now welcoming and equal, some people may have a legacy of their previous negative experiences to counteract. There are interactions with other members of staff and students to contend with. Some colleagues may be overtly sexist or harassing; others may make so-called "joking" comments which still hurt to the bone. There are colleagues who are apathetic - they may feel this is a problem which does not concern them or believe that gender inequalities have already been "fixed". They might wonder what all the fuss is about, or even feel slightly hostile to the whole Athena SWAN agenda because they perceive it as special treatment for 
women at the expense of opportunities for men. A less obvious form of inequality results from benevolent sexism where the colleague is well-intentioned but still treats women differently by patronising them or attempting to protect them. Yet, among this mix of colleagues there are also committed people who have dedicated a huge amount of effort to reducing inequalities and improving the working lives of their colleagues. Such people are often, but not exclusively, to be found on Athena SWAN working groups.

For the many universities in the UK which have teams of colleagues committed to the Athena SWAN and other equality challenges, what is the most effective way for the initial committed few to make lasting changes? Lasting change requires a strong and public university commitment to fully support people who face harassment and a set of clear procedures which make it harder to inadvertently discriminate. A set of procedures by itself is not a complete solution; organisational change also requires changes to people's behaviour. Universities must find ways to guide, encourage and educate a critical mass of colleagues to routinely behave in a way which contributes to a fair and equal working environment. This is not easy to accomplish, given the range of existing attitudes and perspectives among staff and students. But recent research illustrates that it is possible.

In this article and other articles in the book, we describe our understanding of the approaches which universities can take to gender equality with reference to current theory and evidence in behavioural economics, behavioural change and psychology. We have prioritised findings from carefully designed studies of real work environments, and tracked down syntheses and meta-analyses which represent the best evidence available. Our aim has been to find useful, actionable advice which can make positive differences to the working lives of our colleagues, setting it within a theoretically informed framework for practice. Elsewhere in the book you can find contributions from authors, illustrators, and photographers which give a rich layer of personal experience and expertise from those who have disrupted gender inequalities when they found them.

\section{Gender bias as a remediable habit}

A useful way to consider the problem is to regard gender bias as a bad habit which a university is trying to break. Carnes and colleagues suggest that addressing structural issues alone, while necessary, is insufficient to achieve gender equity. They propose that it is also necessary for individuals to change their behaviour, based on an appreciation of their own biases (Carnes et al., 2015). They a pproach gender bias as a remediable habit, and between 2010 and 2012 conducted a cluster randomised controlled trial to gauge the effect of an intervention that sought to break the gender bias habit of faculty in a range of academic departments.

The study involved 2,290 staff members from 92 academic departments at the University of Wisconsin-Madison. Participants in the intervention group took part in a 2.5 hour interactive workshop about reducing gender bias. At a threemonth follow up, in the departments in which $25 \%$ or more of staff attended workshops, there were significant increases in self-reported action to promote gender equity, and staff expressed significantly greater perception of fit within their departments, and greater comfort in raising social or personal issues at work.

The effects sizes ranged from 0.11 to 0.32 , which are small but are in the expected range for behavioural research. This study is instructive in the modesty of the intervention. The fact that improvements took place when only $25 \%$ of staff members of a department attended is encouraging, as is the fact that the workshops did not require a large time commitment for individuals. Future research could unpick how increasing the workshop length or 
coaxing a larger proportion of staff to attend would change the size of the effects, but the wider point is that education for a relatively small proportion of staff appears to make a difference to individuals' experience of the working environment. Further, Carnes et al. note that the perceived improved climate created by their educational and behavioural intervention benefited both male and female faculty (Carnes et al., 2015).

Universities across the UK have already invested in equality and diversity training courses for staff. It is relatively common within Athena SWAN submissions to note that particular groups of staff are required to attend such sessions. Unfortunately, the evidence for the effectiveness of equality and diversity training is generally not compelling (Wilson, 2011; Bohnet, 2016) partly because they are often not evaluated robustly. The work of Carnes and colleagues is important because their evaluation uses rigorous research methods, and the content of the workshop is informed by theory and empirical research (Carnes et al., 2012). We should have a high threshold for the quality of workshops universities require their staff to attend!

The work of Carnes and colleagues is based on a four-stage conceptual model (see Figure 1) charting how faculty, as individuals, moved from being unaware that lack of women in leadership roles is a problem (specifically in departments of academic medicine, science and engineering where the study was focused), through awareness (Stage 1), external motivation (Stage 2), self-efficacy, and expectations of positive outcomes/internal motivation (Stage 3), to action (Stage 4).

\begin{tabular}{|c|c|c|c|}
\hline Awareness & Motivation & \multicolumn{2}{|c|}{$\begin{array}{cc}\text { Self- } & \begin{array}{c}\text { Positive } \\
\text { outcome }\end{array} \\
\text { efficacy } & \text { expectations }\end{array}$} \\
\hline \multirow{2}{*}{$\begin{array}{l}\text { Is unaware that } \\
\text { lack of women } \\
\text { in leadership } \\
\text { in academic } \\
\text { medicine, science, } \\
\text { and engineering } \\
\text { is a problem }\end{array}$} & \multicolumn{3}{|c|}{$\begin{array}{l}\text { To move from motivation "I want to do it" to } \\
\text { action requires both self-efficacy "I can do it" and } \\
\text { positive outcomes expectations "I will benefit from } \\
\text { doing it". To habitually change behaviour requires } \\
\text { deliberate practice of the desired behaviour. }\end{array}$} \\
\hline & $\begin{array}{l}\text { Acts without } \\
\text { bias to avoid } \\
\text { appearing } \\
\text { prejudiced to } \\
\text { others (external } \\
\text { motivation) } \\
\text { Is bias illiterate } \\
\text { May be sincere in } \\
\text { desire for equity } \\
\text { but cognitive } \\
\text { processes lead to } \\
\text { biased behaviour }\end{array}$ & $\begin{array}{l}\text { Acquires } \\
\text { knowledge } \\
\text { and skills, feels } \\
\text { confident in } \\
\text { applying them, } \\
\text { and desires } \\
\text { the outcomes } \\
\text { of promoting } \\
\text { gender equity } \\
\text { in academic } \\
\text { medicine, science, } \\
\text { and engineering }\end{array}$ & $\begin{array}{l}\text { Is internally } \\
\text { motivated to } \\
\text { habitually, } \\
\text { unconsciously act } \\
\text { without gender } \\
\text { bias in academic } \\
\text { settings } \\
\text { Is bias literate } \\
\text { Attends to cues } \\
\text { for bias reduction } \\
\text { strategies } \\
\text { Automatically } \\
\text { acts to prevent } \\
\text { gender bias from } \\
\text { emerging }\end{array}$ \\
\hline
\end{tabular}

Figure 1. Conceptual model underpinning multistep process for reducing gender bias (Carnes et al 2015; adapted with authors' permission) 
They say:

To move from motivation (I want to do it) to action requires both selfefficacy (I can do it) and positive outcome expectations (I will benefit from doing it). To habitually change behaviour requires deliberate practice of the desired behaviour. (Carnes et al., 2015, p. 222)

Approaching gender bias as a remediable habit rather than a 'bad thing which makes me a bad person' removes blame and defensiveness, opening up possibilities of behavioural change through changed practices. In Carnes et al's research intervention ('gender-bias-habit-changing' workshops) participants first identified their own biases, then replaced them with deliberate practice of non-biased behaviour, motivated externally (Stage 2) and then internally (Stage 3) until the new behaviours became habitual (Stage 4).

\section{Stage 1: Awareness}

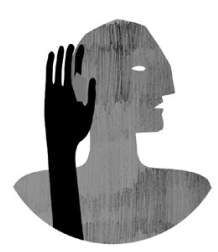

At the start of the first stage, staff may be unaware of the extent of the inequalities which women still face, and might (consciously or otherwise) assume that

there are proportionately fewer women leaders because they lack leadership skills, or because they would prefer not to be leaders, or that being an academic is incompatible with being a mother or that it is a pipeline problem which will eventually resolve itself. Accurate information and education can raise an individual's awareness of a problem, but they may require an additional external motivator to accept the responsibility to take action to change their habits.

Recent publications in high-profile journals which have drawn attention to the extent of gender bias in academia are a great contribution to convincing people that action should be taken (Bedi et al., 2012; Eagly \& Miller, 2016; Lerback,
2017). Athena SWAN has worked well to propel people within UK universities along the behavioural change stages. The requirement to benchmark data for an Athena award raises awareness of the scale of problems with inequality in the proportions of women staff or students.

\section{Stage 2: External motivation}

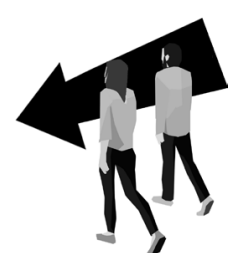

Individuals at this stage in Carnes' model, may be sincere in their desire for equity, but be "bias illiterate" i.e. unfamiliar with the cognitive biases which are part and parcel of our human psychology, regardless of gender (see Unconscious bias for a further discussion of these). At this stage, motivation to reduce gender bias comes from external sources, including the desire to avoid appearing biased to others.

At the very least, senior management are under some pressure to commit their universities to Athena SWAN principles because they do not wish the institution to appear prejudiced. At the level of interpersonal interactions, people begin to realise that certain words and deeds are not acceptable to others and modify their behaviours. The pressure from Athena SWAN to shift towards more equal numbers of women, particularly in promoted posts, drives departments to change their recruitment and promotion procedures. Such human resource policies are also external signposts to routinely behave in a non-biased way.

Research from behavioural economics can inform us about effective ways to design procedures to act as external motivators for non-biased behaviour on the part of individuals. Iris Bohnet's book What Works: Gender Equality By Design (Bohnet, 2016) suggests easy, low cost but surprisingly effective changes to the work environment which might help to reduce gender inequalities. The goal of behavioural design is to nudge and shift processes used by 
organisations so that they are likely to yield fairer results. Bohnet (2016) is a carefully researched synthesis of meta-analyses and empirical work relating to a range of topics including staff recruitment, worklife interactions, working in groups and interactions between colleagues at different career stages. Bohnet introduces the mnemonic DESIGN - Design, Experiment, SIGN. That is, organisations should (re) design a fairer process, experiment to see if it is effective and then signpost that design to ensure people use it. The signposts should point us in a direction where we can easily make unbiased choices. Bohnet takes the view that one should not try to change minds, but rather use signposts - "the very purpose of signposts is to help us find the way without having to memorise or even think much about it".

An integral part of this approach is to gather benchmark data to make it possible to evaluate and refine designs. For example, the Athena SWAN Bronze award requires applicants to gather benchmark data sets on student applications and acceptances as well as staff at various grades. The accompanying action plan is a design for improving the benchmarks.

In the EqualBITE team, we are open to behavioural design strategies, but we also believe that we should try to change attitudes particularly in the University environment. As educators, we are in a powerful position to persuade our students of the moral importance of equality and encourage them to act to promote inclusion in their future lives. This brings us to the next stage of Carnes' model where external motivation to act becomes internalised.

\section{Stage 3: Self-efficacy and positive outcome expectations}

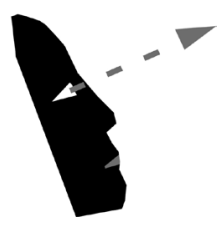

Those at stage 3 in the conceptual model have acquired the knowledge and skills to replace their previously biased habits with less-biased behaviours. They have the confidence to apply their knowledge, and desire the outcome of gender equality.

Carnes and colleagues provide five evidence-based strategies for workshop participants to practise, and two counterproductive strategies to avoid. Bias can be overcome by a) stereotype replacement where an individual notices that they have fallen into the trap of a stereotype and consciously replaces that stereotype with more accurate information; b) positive counter-stereotype imaging through which a person imagines an effective women leader in detail; c) perspective taking during which a person imagines what it would be like to be a member of a minority group; d) individuation where the person replaces assumptions about a student or a job applicant with specific factual information and e) routinely interacting with successful women who run counter to stereotype. They note that attempting to suppress stereotypes or holding a strong conviction that one is bias-free are not likely to be effective at kicking the gender bias habit. Workshop participants become confident at using these strategies over time with deliberate practice - which is often the key to successful behavioural change.

However, before an individual goes to the mental effort of applying these strategies, they have to believe doing so will have a positive outcome. The slogan we used to recruit people to our early recipe-writing workshops was "When it's better for women, it's better for everyone" by which we mean that warming up a chilly climate makes all staff members' lives easier.

Everyone benefits from courteous behaviour, transparent promotion procedures and flexible working arrangements. But clearly not everyone directly benefits from womenonly initiatives such as women's networking groups or scholarships for women. Such opportunities can be resented by men (Fox, 2014) particularly if they view academic life as a zero-sum game in which wins for a female colleague necessitate losses for 
them. It is true that some areas of academic life-such as research funding - are fiercely competitive. Other areas are not - since the advent of online publishing it would appear there is no end to the journal articles which the world can absorb. At any rate, in our view, initiatives which target women or minority groups are a small step towards rebalancing the scales of opportunity.

\section{Stage 4: Action}

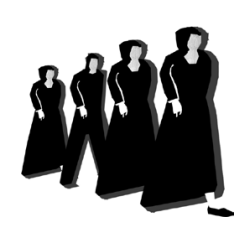

People in stage 4 of the conceptual model habitually act in a way to reduce bias, are bias literate and notice when bias-reduction strategies are needed. Having reached this state of enlightenment, they are then ideally placed to assist other people on their own quests. Currently, it may be possible to find such people on Athena SWAN self-assessment teams, or in the Equality and Diversity section of Human Resources. However, the longer term goal is for everyone to gradually move towards this stage. The higher the proportion of individuals within a department at stage 4 in the conceptual model, the more likely it is that everyone in that department will find the working environment positive.

We aspire to a university where people in teaching roles such as lecturer, personal tutor or programme director have also reached stage 4 and make teaching decisions accordingly. If we want more women and members of minority groups to choose to study and thrive in disciplines traditionally dominated by white men, teaching staff need the skills, confidence and pedagogical knowledge to create supportive learning environments in every class. We know that being exposed to stereotype threat and having experiences of working or studying within a chilly climate can prevent individuals from reaching their potential. There is evidence that these psychological circumstances can impair people's performance and reduce their confidence and ambition (Steele, 2010).
If teaching staff were aware of the literature on 'wise psychological interventions' in educational settings (Walton, 2014) and routinely applied this knowledge with their students, classrooms would be warmer for all learners, and some of the consequences of stereotype threat which students may have previously experienced could be mitigated. Wise psychological interventions attempt to change the attitudes, narratives and mindsets within individuals. They are based on specific, precise well-founded psychological theories which have been validated in the lab and then developed to be applicable in real world settings. The reason that small interventions of this sort can have surprisingly long-lasting effects is that they operate recursively. A small adjustment to the way an individual perceives their situation (when applied early enough) can disrupt a self-reinforcing downward spiral, and begin a snowball of positive effects. See Stereotype threat for further explanation of how wise psychological interventions can be applied to teaching.

\section{The role of senior leadership within the institution}

\section{Representation and inclusion}

Lumby (2009) examines how an institution's approach to diversity is informed by senior leaders' different value sets. She uses two case studies (a community college and a sixth form college) to explore how leadership approaches and values relate to how the colleges tackled diversity issues (gender as well as ethnicity, age and dis/ability).

Through interviews and surveys, Lumby asked: a) how senior leaders understood diversity; b) what, if any, did they see as their goals; c) what the leaders believed to be pressure(s) for action or inhibitor(s) of action, and what degree of pressure they experienced; and d) what action, if any, resulted (Lumby, 2009, p. 428). From the data, she identified two main leadership approaches: leading for and leading with diversity in which: 
Leading for diversity essentially targets the injection of more people from under-represented groups into leadership positions. Its goal is representation. [...] Leading with diversity aims to achieve structures and a culture which are equally supportive of all so that people, whatever their nature or background, can work productively while remaining true to themselves. (Lumby, 2009, pp. 441-442)

From Lumby's perspective, achieving equality in education is not just about achieving representative or equal proportions of people with particular protected characteristics in a department. It is about creating a working culture which thrives on diversity. Leaders should recognise the importance of welcoming new staff and students into the culture and building on their strengths, rather than asking them to work in the style of the dominant group.

Lumby posits a four-box model where high and low importance is given by leaders to representation (y axis) and inclusivity (x axis) (see Figure 2).

To some extent this can usefully be mapped onto Carnes et al's stages as part of systemic change drivers. Thus, as individuals and leaders develop their own bias awareness and make a shift in their espoused values, their departments can move from compliance with gender equality regulations, then to ensuring a more equal gender representation (working for diversity), next to creating a culture of inclusivity that in turn, finally becomes systemic (working with diversity) as part of what Mackay (2014) calls the 'institutional blueprint'.

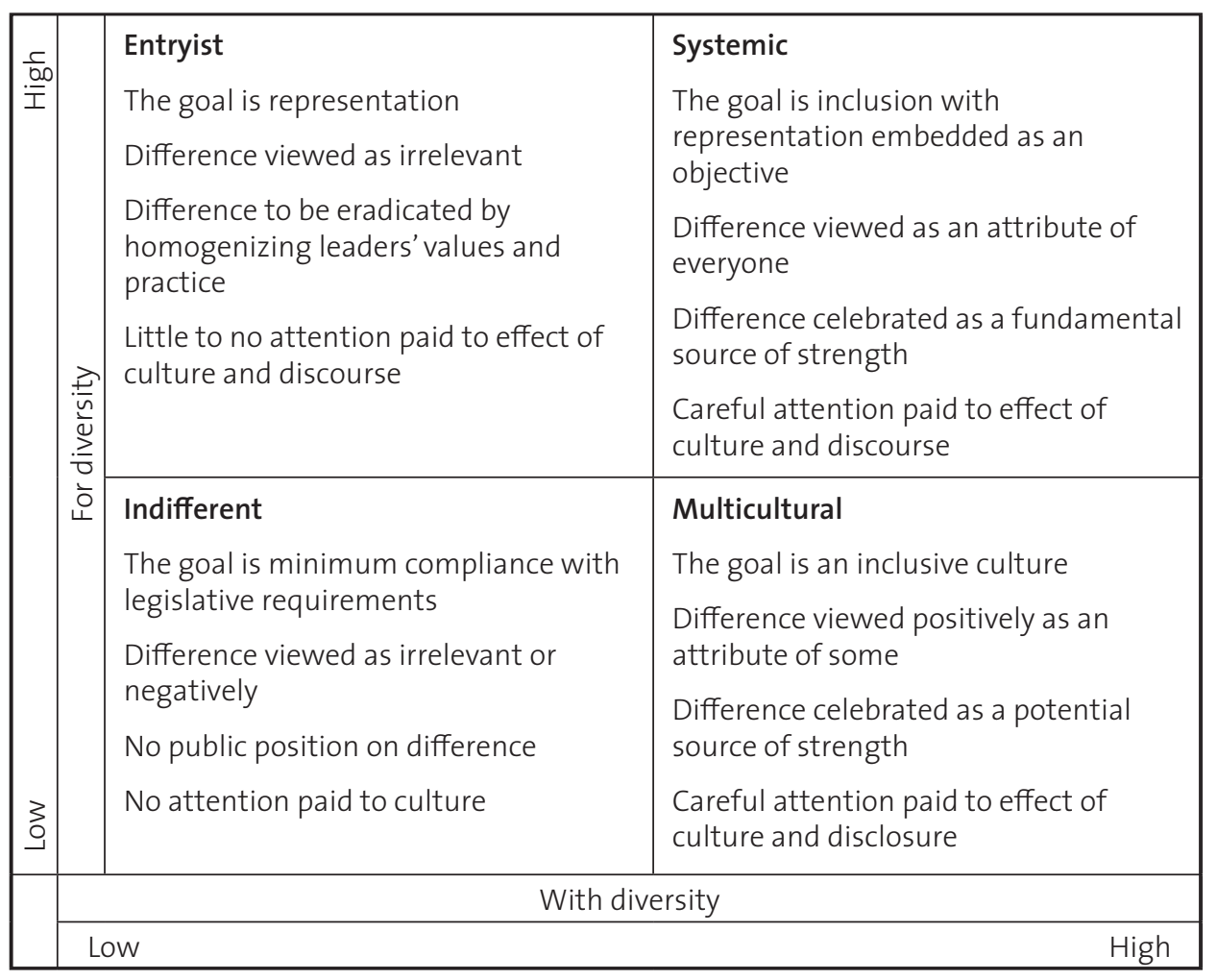

Figure 2. A model of orientations to diversity (Lumby 2009) reproduced with author's permission 
It is worth noting, however, that the Carnes model is focused on removing bias without necessarily promoting an inclusive culture. Thus a leader who has reached Stage 4 of the Carnes model may highly value working for diversity, and habitually act to do so without also promoting inclusivity and the celebration of difference. We agree with Lumby that it is important to value both representation and inclusion.

\section{Sustaining change}

Mackay (2014) sets out the difficulties encountered in embedding gender reforms:

The central findings of empirical research highlight variable outcomes across cases and the coexistence of elements of continuity and change and caution that change in one institutional arena may be supported or confounded by the effects of other institutional arenas, illuminating the difficulties encountered in embedding gender reforms (see, for example, Chappell 2011; 2014; Goetz 2003; Kenny 2013; Majic 2014; Waylen 2007). (Mackay, 2014, p. 550)

As part of the EqualBITE project, we invited senior University leaders to reflect on the key issues about gender equality as they saw them (see Leadership perspectives on gender equality). They spoke of the

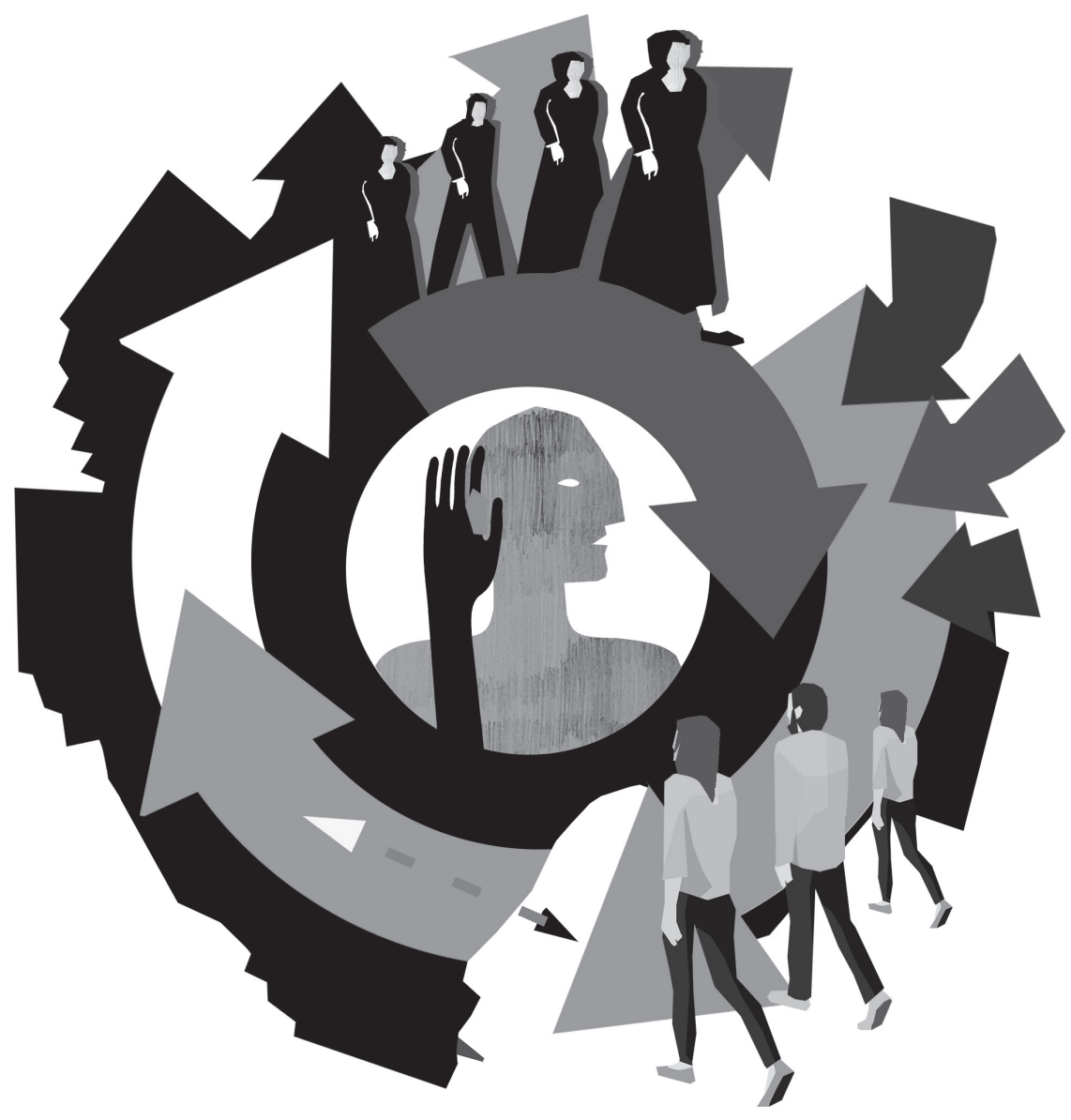


"significant structural barriers" to gender equality that could only be tackled through "a systems level change".

The senior leaders' conversations acknowledged the need to continue to "work within the institution" to support and maintain the "fragile new power structures" and the progress already made, and the particular "need to be careful we don't revert back". Mackay identifies "two mechanisms through which institutional innovation is actively resisted or passively neglected: 'remembering the old' and 'forgetting the new'" and argues that:

The stickiness of old rules (formal and informal) about gender, the "nestedness" of new institutions within the wider environment, and the way newness functions as a gendered liability provides a powerful explanation for why it is so hard to make gender reforms - and wider institutional change conducive to the regendering of politics - stick. (Mackay, 2014, pp. 550-551).

A leader in Carnes et al's fourth stage, will habitually and automatically act to reduce bias when she encounters it; that is, she remembers the new. Old rules about gender are seen as a habitual way of thinking; habits embodied in the old rules can be systematically replaced by new non-biased rules. It may take some time before the old habit has completely gone, and the temptation to return to a newly broken habit may be strong at times of stress, or when surrounded by people who still enjoy the old habit. Despite these potential setbacks, it is entirely possible to change behaviour, and as the work of Carnes et al. demonstrates, useful results can be achieved when a relatively low proportion of staff commit a small amount of time to changing their habits of thought. A future study on the impact of remediating the gender bias habit in senior leaders would be instructive: to what extent do changes in the attitudes of leaders impact the day-to-day experiences of staff? Do staff have higher work satisfaction when their leaders embrace inclusivity as well as representativeness?

\section{Conclusions}

We believe that achieving gender equality is such a challenging task that it needs to be tackled on several fronts. We need institutions to remove barriers and facilitate structural change; behavioural design of procedures and processes can assist with this. This will not be sufficient on its own - a productive working and learning environment requires people to treat each other equally and with respect. If we consider gender bias as a habit which can be broken, then carefully applied strategies for behavioural change will help. For those individuals who have already experienced the negative consequences of bias or stereotype threat, insights from wise psychological interventions may be useful. Leaders who habitually act to reduce bias, who celebrate inclusivity and see strength in differences will also help to achieve institutional change.

However, we believe that we should not stop with changes within the academy. At the heart of the EqualBITE project is the conviction that universities can - and should - be beacons of gender equality, acting as agents of change, building a society of gender equality, and enabling change in the attitudes and behaviours of the people who work, study and research within them. While change can begin within the walls of ivory towers, we would like our graduates to use their experiences of learning in an inclusive university to challenge the inequalities they meet in wider society. 